\title{
UK guidance for the pathological reporting of serrated lesions of the colorectum
}

\author{
Adrian C Bateman, ${ }^{1}$ Neil A Shepherd ${ }^{2}$
}

${ }^{1}$ Department of Cellular Pathology, Southampton General Hospital, Southampton, UK ${ }^{2}$ Gloucestershire Cellular Pathology Laboratory, Cheltenham General Hospital, Cheltenham, UK

\section{Correspondence to} Dr Adrian C Bateman, Department of Cellular Pathology, MP002, Level E, South Block, Southampton General Hospital, Tremona Road, Southampton S016 6YD, UK;

adrian.bateman@uhs.nhs.uk

Received 12 March 2015 Revised 7 April 2015 Accepted 13 April 2015 Published Online First 1 May 2015

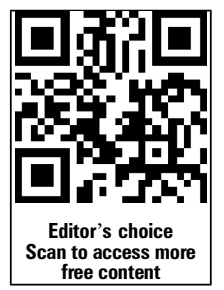

\section{CrossMark}

To cite: Bateman $A C$, Shepherd NA. J Clin Pathol 2015;68:585-591.

\section{ABSTRACT}

Bowel cancer screening programmes have highlighted to endoscopists and clinicians the spectrum of serrated colorectal lesions. One of the most significant developments has been the recognition that sessile serrated lesions (SSLS), while bearing histological resemblance to hyperplastic polyps (HPs), may be associated with the enhanced development of epithelial dysplasia and colorectal adenocarcinoma. Different minimum criteria exist for the diagnosis of SSLS and their differentiation from HPs. Furthermore, the spectrum of terminology used to describe the entire range of serrated lesions is wide. This variability has impaired interobserver agreement during their histopathological assessment. Here, we provide guidance for the histopathological reporting of serrated lesions, including a simplified nomenclature system. Essentially, we recommend use of the following terms: HP, SSL, SSL with dysplasia, traditional serrated adenoma (TSA) and mixed polyp. It is hoped that this standardisation of nomenclature will facilitate studies of the biological significance of serrated lesions in terms of the relative risk of disease progression.

\section{INTRODUCTION}

Bowel cancer screening programmes have highlighted the histopathological assessment of serrated colorectal lesions as a problematic area. The terminology used to describe lesions within this spectrum is variable and the suggested minimum diagnostic criteria for some lesions differ between authorities. ${ }^{1}$ This has led to poor interobserver agreement during the histopathological assessment of this range of entities. ${ }^{2-4}$ One of the most difficult areas is the nomenclature of and diagnostic criteria for sessile serrated lesions (SSLs) (also termed 'sessile serrated adenoma' (SSA) or 'SSA/polyp' (SSA/P)). This is particularly important as these lesions, while bearing histological resemblance to hyperplastic polyps (HPs), may be associated with the early development of epithelial dysplasia and colorectal adenocarcinoma. ${ }^{5} 6$ Therefore, distinction of these lesions from HPs-that are associated with little or no increase in colorectal cancer riskis very important. However, application of different diagnostic criteria may lead to differing thresholds for a diagnosis of SSL between reporting histopathologists. The levels of risk of disease progression associated with lesions diagnosed using these differing criteria are unclear. Variations in the application of terminology may also result in the same lesion being afforded different names by reporting pathologists. This can lead to confusion among clinical teams managing these patients and may inhibit studies of the biological significance of these lesions and the risk of disease progression associated with various histopathological factors.

This review provides guidance relating to a simplified nomenclature and classification system for serrated colorectal lesions.

\section{NOMENCLATURE}

The nomenclature of serrated lesions is complex, and there are differences of opinion between UK, European and US pathologists regarding the optimal terminology. We propose that serrated lesions should be given one of the following names according to their morphological features: HP, SSL, SSL with dysplasia, traditional serrated adenoma (TSA) and mixed polyp (box 1). The definitions of these lesions are given below.

\section{Hyperplastic polyp}

These are small serrated lesions showing no features that would allow categorisation as an SSL and no evidence of dysplasia. We use the term 'dysplasia' in this context to refer to the morphological appearances of epithelial neoplasia within the mucosa of the colon and rectum, for example, the epithelial changes recognised by histopathologists as characteristic of 'classical' adenomas. HPs are usually small (less than $5 \mathrm{~mm}$ diameter) and may occur anywhere within the colon and the rectum. However, they are particularly common in the distal colon and rectum and are often multiple. It is recognised that there are two common morphological forms of HPs. ${ }^{7}$ The slightly more common histological form is the microvesicular HP. They demonstrate vesicular mucin-containing epithelial cells (figure $1 G$ and $1 \mathrm{H})$. Goblet cells are decreased in number compared with normal crypts. Goblet-cell-rich HPs account for about one-third of all HPs, and these too almost always occur in the left colon and rectum. Unsurprisingly, given their name, they show numerous goblet cells. Microvesicular HPs tend to demonstrate $B R A F$ mutations whereas KRAS mutations are predominant in the goblet-cell-rich variant. There is also a 'mucin-poor' variant, but this is rare. This type is similar to microvesicular HPs, but contains less microvesicular mucin and less goblet cells.

We believe that the risk associated with small HPs is very low. They are particularly common in the distal colon and rectum. Although there is some evidence to suggest that HPs of the left colon and rectum may be part of the serrated neoplasia pathway that ultimately leads to left-sided serratedtype carcinoma, especially on molecular evidence, ${ }^{8}$ it is likely that the progression of HPs to SSLs takes a considerable time and, especially in older patients with only a few HPs and in patients who have bowel cancer screening, the risk of progression in 
Box 1 Recommended terminology for (non-invasive)

serrated lesions of the colon and rectum

- Hyperplastic polyp (HP)

- Sessile serrated lesion (SSL)

- SSL with dysplasia

- Traditional serrated adenoma (TSA)

- Mixed polyp

their lifetime is very low. The condition once termed 'hyperplastic polyposis' is now known as 'serrated polyposis' as it is recognised that the index mucosal lesion in this condition shows features that are different to the 'classical' HP, and this is now termed either an 'SSA/P' (North America) $)^{5}$ or an SSL (UK).

\section{Sessile serrated lesion}

These lesions are referred to as 'SSA' or 'SSA/P' in North America. When 'pure', these lesions show no evidence of dysplasia, but in comparison with HPs, they contain one or more of the histopathological features listed in box 2 (figure 1A-1F). No 'ranking order' for these features has yet been established. SSLs also show 'dysmaturation', that is, a disorganised arrangement of proliferating cells and goblet cells within the lower half of the crypts, with subtle cytological abnormalities that are more pronounced than in HPs. Some pathologists believe that 'dysmaturation' represents a form of dysplasia, but these changes are distinct from those that are recognised as dysplasia within 'classical' adenomas. In the UK, we firmly believe that 'adenoma' is an inappropriate terminology for a lesion in which morphological dysplasia is not demonstrable, and hence, we

Box 2 Key histological features of sessile serrated lesions (SSL)

- Irregular distribution of crypts

- Dilatation of crypt bases

- Serration present at crypt bases

- Branched crypts

- Horizontal extension of crypt bases*

- Dysmaturation of cryptst

- Herniation of crypts through muscularis mucosa

- WHO criteria: at least three crypts or at least two adjacent crypts must show one or more of these features to enable a diagnosis of SSL

- American Gastroenterology Association criteria: one crypt showing the characteristic features is sufficient for the diagnosis of SSL

Key: *Involved crypts often have an ' $L$ ' or inverted ' $T$ ' shape. tDysmaturation is disordered cellular maturation within crypts and is evidenced by subtle nuclear enlargement, crowding, pseudostratification and mitotic activity together with the presence of a disorganised mixture of non-mucus-containing epithelial cells and mature goblet cells within the deep aspects of crypts. In this context, assessment of proliferation index, for example, using MIB-1 may provide supporting evidence for a diagnosis of SSL by highlighting epithelial cell proliferation within the superficial half of crypts. However, such immunohistochemistry, while sometimes helpful, does not reveal features that are alone diagnostic of SSL. would not use the term SSA for such a lesion. Critically, the minimum criteria required for a diagnosis of SSL is still under debate. For example, the WHO classification recommends that this diagnosis should be made when three crypts within a lesion -or two adjacent crypts-show at least one of the characteristic features. ${ }^{9}$ However, the American Gastroenterology Association recommends that the diagnosis should be made even when a single crypt contains one or more of the characteristic features. ${ }^{10}$ SSLs may be of any size, but are commonly $10 \mathrm{~mm}$ or more in diameter. Of these, we recommend use of the WHO criteria. Lesions within the HP-SSL spectrum over $10 \mathrm{~mm}$ in diameter will almost certainly contain at least three-or two adjacent-crypts showing the characteristic features of SSLs, anyway.

HPs and SSLs share many morphological features and both are associated with mutations in the BRAF gene. ${ }^{11}$ It is therefore possible that they represent part of the same 'spectrum' of serrated lesions, with small HPs at one end and larger $(10 \mathrm{~mm}+)$ SSLs at the other. The larger lesions that are classified as SSLs usually contain several crypts showing one or more of the 'characteristic' features of SSLs, while smaller lesions (typically $5-10 \mathrm{~mm}$, but sometimes less than $5 \mathrm{~mm}$ ) may contain a single crypt-or a very small number of crypts-with the 'characteristic' SSL features. According to which guidelines are followed, such polyps may or may not be diagnosed as SSLs. However, this spectrum of features is an interesting 'unifying hypothesis' for the relationship between HPs and SSLs. Indeed, the condition originally termed 'hyperplastic polyposis' is now termed 'serrated polyposis' after the morphological features of SSLs were identified in this setting. ${ }^{5}$ In this model, it is unclear why tiny, often distal, HPs do not appear to be associated with a significant risk of colorectal cancer development, while larger lesions with the features of SSLs can be associated with the development of dysplasia and adenocarcinoma.

\section{SSL with dysplasia}

SSLs may contain a focus of dysplasia as defined in the previous section. This dysplasia may be low or high grade in nature and is almost invariably present within a lesion that also shows features of an SSL without dysplasia. It is believed that these lesions may particularly be associated with faster progression to adenocarcinoma than 'classical' adenomas. The term 'mixed polyp' has also been used to describe this lesion. Dysplasia arising in the context of an SSL commonly shows loss of the DNA mismatch repair enzyme, hMLH-1, as part of a genetic signature that includes $B R A F$ mutation and widespread DNA methylation (the 'CpG island methylator phenotype') (figure $2 \mathrm{~A}-2 \mathrm{G}$ ). ${ }^{7}$ We believe that most mixed polyps, especially in the right colon, represent various stages in the serrated neoplasia pathway, namely the presence of an SSL within which dysplasia has arisen. Terms that have been used to describe this lesion include 'SSL with cytological dysplasia' (an adaptation of the current WHO classification 'SSA/P with cytological dysplasia') and 'SSL with conventional dysplasia'. Given that UK pathologists would not recognise the 'dysmaturation' present within uncomplicated SSLs as 'true' dysplasia, we believe that the simpler term 'SSL with dysplasia' describes these lesions most accurately and succinctly.

\section{Identification of lesions within the HP-SSL spectrum with the highest risk of disease progression}

There are currently no data that clearly indicate the risk of development of colorectal cancer associated with the finding of an SSL or how this risk might relate to the minimum diagnostic 
criteria used and the lesion size. However, it is commonly believed that larger lesions and those showing dysplasia are associated with the greatest chance of disease progression.

\section{Reproducibility of diagnosis of lesions within the HP-SSL spectrum}

The SSL is a relatively newly described entity, and its reliable distinction from the HP can still be problematic. Pathologists are gradually becoming aware of the SSL as a lesion that is important in the development of colorectal cancer, ${ }^{12}$ as well as the constellation of histological features seen within SSLs. However, recent studies have suggested that SSL is still an underdiagnosed entity and that significant interobserver variability exists during the assessment of these lesions. ${ }^{2-4}$ A key difficulty is that the minimum criteria for the diagnosis of SSL have varied between guidelines and between groups of pathologists. ${ }^{9}{ }^{10}$ Also, the small size of some biopsies and lesions as well as difficulties associated with malorientation of biopsy fragments can preclude definitive distinction between HPs and SSLs in some cases. There is evidence that the use of a reference article on diagnostic criteria can improve diagnostic reproducibility in serrated lesions. ${ }^{13}$ Furthermore, while SSLs have been linked to the development of colorectal cancer, possibly via a molecular pathway that proceeds more rapidly than the classical Vogelstein model, ${ }^{14}$ the magnitude of this risk has not yet been clearly stratified according to the minimum criteria used for diagnosis or to other features of the lesions such as their size and location.

Although not definitive, one association with the type of serrated pathology present is the anatomical site. Although lesions
Figure 1 Features of sessile serrated lesions (SSLs) and a hyperplastic polyp (HP). (A-G) SSLs. (A and B) Crypt dilatation, usually particularly evident in the lower half of the crypt. (C) Pronounced serration in contrast to the gentle epithelial undulation that is characteristic of traditional serrated adenomas (TSAs). This feature may be particularly widespread in some SSLS. (D) Horizontal spreading of crypts, forming an ' $L$ ' shape in this example. (E) Serration within the basal aspect of a crypt. This feature may not be evident in SSLs that show basal crypt dilatation. (F) Herniation of crypts through the muscularis mucosa. This is not a particularly common feature and is usually focal. (G) A well-orientated lesion, in which crypts are present in longitudinal section. Distinction from an HP in this situation is more straightforward than when a lesion is sectioned tangentially or parallel to the mucosal surface. (H) A microvesicular HP showing serration within the upper half of the lesion and without any of the characteristic features of SSLS $(A-D, F$ and $H$ ) magnification $\times 100$; (E) magnification $\times 200$; $(\mathrm{G})$ magnification $\times 40$.
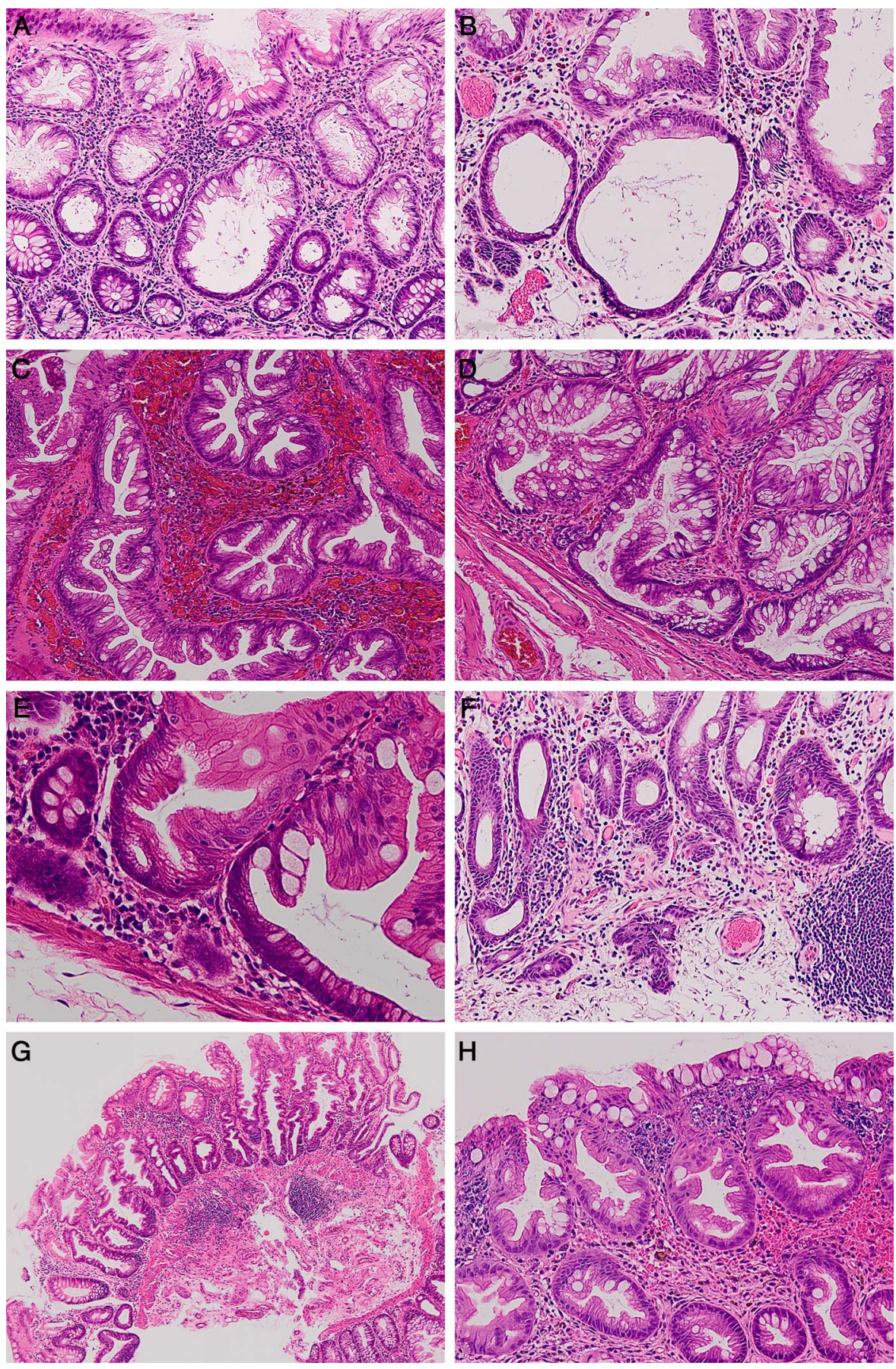
Figure 2 Sessile serrated lesions (SSLs) with dysplasia. (A and B) An SSL showing a sharp distinction between areas showing no dysplasia and low-grade dysplasia. (C and D) Immunohistochemistry on the same lesion as within (A and $B$ ), showing loss of nuclear hMLH-1 expression within the crypts with low-grade dysplasia. Expression of hMLH-1 is preserved within stromal and inflammatory cells (C) and within non-dysplastic crypts (D). (E and F) Another SSL showing areas without dysplasia (E) and with low-grade dysplasia (F) that is more subtle within the lesion illustrated in A-D. (G) Immunohistochemistry on the same lesion as within $E$ and $F$, showing loss of nuclear hMLH-1 expression within the area of dysplasia and therefore helping to highlight this feature. Expression of hMLH-1 was retained within the non-dysplastic crypts. $(\mathrm{H})$ A 'classical' tubulovillous adenoma showing ectopic crypt foci, as also seen in traditional serrated adenomas (TSAs). However, no other features of TSAs are present-ectopic crypt foci are characteristic of, but not specific for, TSAs. Compare these features with those of the TSA in figure $3(A$ and $C)$ magnification $\times 100$; $(B, E-H)$ magnification $\times 200$.
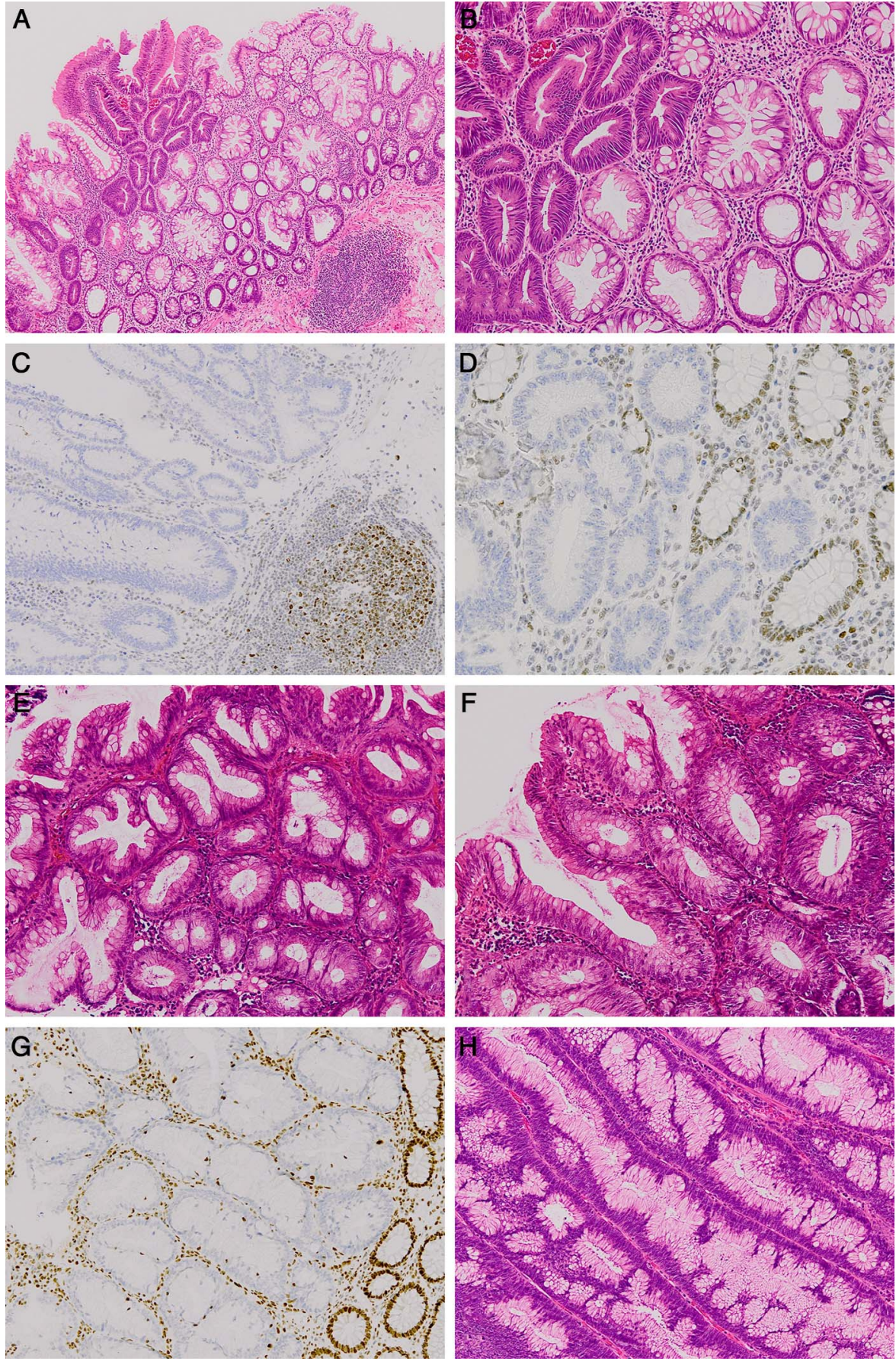

very similar or identical to SSLs may be demonstrated in the left colon and rectum, the great majority of such lesions are found in the right colon. In contrast, HPs and TSAs are preferentially seen in the left colon and rectum. Therefore, an additional level of certainty is required before making a diagnosis of SSL within the left colon. The use of size and location data may improve interobserver agreement in the diagnosis of these lesions. ${ }^{15} 16$ Identification of dysplasia within an SSL (ie, 'an SSL with dysplasia') is likely to create less diagnostic difficulty than the distinction between (some) SSLs and HPs, in that pathologists are used to identifying dysplasia in gastrointestinal mucosal biopsies. Immunohistochemistry can help to highlight dysplasia in this setting, since its development may be associated with loss of DNA mismatch repair enzyme expression, especially hMLH-1 and PMS-2.

\section{Traditional serrated adenoma}

These lesions are distinct from SSLs. They most commonly occur in the left colon and rectum and may have a pronounced villiform or even filiform architecture. ${ }^{17}$ They are characterised by the presence of dysplasia (often subtle) together with a variable proportion of the lesion showing eosinophilic cytoplasm, pencillate nuclei and ectopic crypts (figure $2 \mathrm{H}$, figure $3 \mathrm{~A}-3 \mathrm{D}$ ). The serration in TSAs is imparted by a combination of undulations in the crypt epithelium and crypt budding, while that of the SSL is formed by a sawtooth-like arrangement of the crypt epithelial cells without budding. TSAs almost always comprise a mixture of foci showing the above characteristic features, with areas showing a 'classical' adenoma growth pattern, in which obvious dysplasia is present. The proportion of areas showing the characteristic TSA features and 'classical' adenoma features 
Figure 3 Features of a traditional serrated adenoma (TSA) and an example of a 'mixed polyp'. (A-D) A TSA showing a pronounced villous growth pattern in areas $(A)$, pencillate nuclei (especially in A-left side), eosinophilic cytoplasm and ectopic crypt foci (especially in C). Foci of subtle dysplasia that is characteristic of the areas with eosinophilic cytoplasm and pencillate nuclei are mixed with classical low-grade dysplasia (especially in B and C) and with classical high-grade dysplasia (D). The cut-off between the areas of subtle and classical dysplasia is often abrupt. (E-H) A 'mixed' polyp, containing SSL-like areas (E and F), TSA-like areas with subtle dysplasia $(\mathrm{G})$ and classical low-grade dysplasia (H). $\mathrm{G}$ also shows TSA-like undulation of the crypt epithelium, imparting a gentler variant of serration to the architecture than is usually seen in SSLs. Other mixed polyps may comprise hyperplastic polyp (HP) and classical adenoma areas, arranged in such a way so as to give the impression of a 'collision' tumour (all magnification $\times 200$ ).
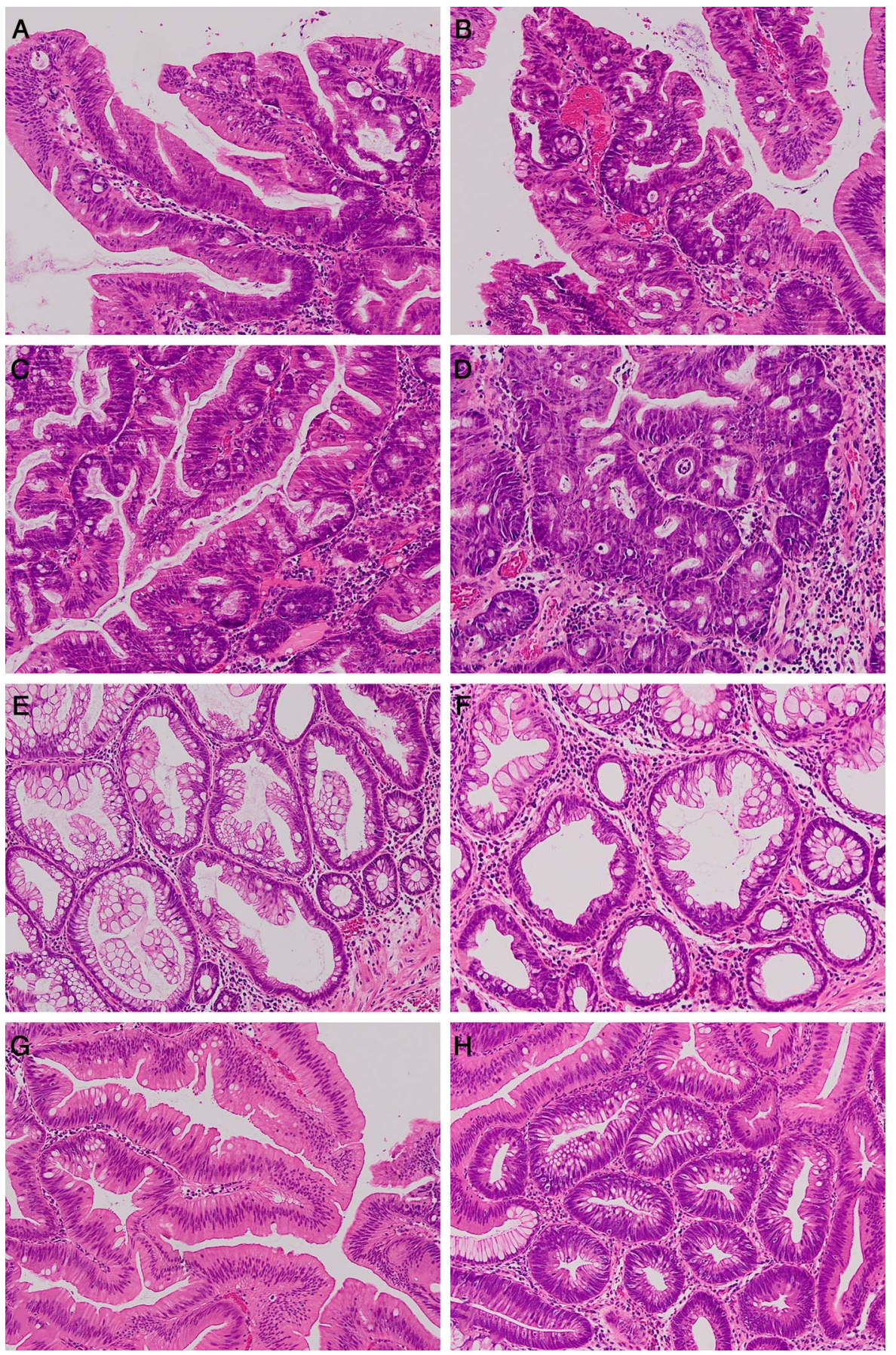

is variable, and the minimum criteria for a diagnosis of TSA are not well defined. However, when assessed even at low or medium power examination, these lesions are much more obviously adenomatous in nature than SSLs. Molecular analysis has revealed that TSAs more commonly possess KRAS mutations and less commonly harbour BRAF mutations than SSLs. ${ }^{11}$ For these reasons, TSAs appear to be more closely related to 'classical' adenomas than SSLs. It therefore seems appropriate that they are managed in the same way as 'classical' adenomas.

TSAs are characterised by a disruption of the signalling pathways involved in stem cell control and cell fate determination. This results in the expansion of a progenitor cell population from the crypt base into the ectopic crypt foci or lateral buds that morphologically characterise this condition. These progenitor cells actively proliferate and accumulate somatic mutations with resultant dysplasia arising from outside of the crypt base stem cell niche. ${ }^{18}$ This evidence underpins, likely, why TSAs seem to have a more rapid malignant potential as the ectopic crypt foci/lateral buds act like additional crypt cell niches and are subject to additional mutations, resulting in more rapid transformation compared with 'standard' adenomas.

\section{Mixed polyps}

While it is our opinion that the majority of 'mixed' polyps, especially in the right colon, represent SSLs with and without dysplasia, we accept that polyps may rarely be encountered, particularly in the left colon, that appear more likely to have arisen due to a 'true' collision event between an HP and a 'classical' adenoma. Furthermore, TSAs are not uncommonly encountered in which a significant component shows the 
features of a 'classical' adenoma. The minimum proportion of a TSA that is required to show features of a 'classical' adenoma in order for the polyp as a whole to be considered 'mixed' has yet to be defined. Furthermore, occasionally, polyps showing a combination of SSL and TSA-like features are encountered, with or without areas with a 'classical' adenoma appearance (figure $3 \mathrm{E}-3 \mathrm{H}$ ). Another variant of the mixed polyp is the combination of HP changes and serrated low-grade dysplasia with features of a TSA. These lesions are more unusual and are seen usually in the sigmoid colon and rectum. While a collision lesion is possible, we believe that the latter mixed polyps usually represent different stages in the traditional serrated neoplasia sequence with serrated dysplasia deriving from a preexisting HP. Due to the existence of lesions such as these, we believe, it is sensible to retain the term 'mixed polyp' within the recommended terminology list, even if it manifests that they may represent different serrated entities and different serrated neoplasia pathways.

\section{Serration in other situations}

It is now recognised that serration may be seen as a complication of chronic inflammatory bowel disease. ${ }^{19}$ The significance of isolated epithelial serration in ulcerative colitis, in particular, is currently uncertain. Serration may also be seen in dysplasia arising in the context of inflammatory bowel disease, for instance within dysplasia-associated lesions or masses.

Furthermore, it would appear that epithelial serration, in the colon and rectum, can occur as a secondary phenomenon. This is particularly seen in stromal lesions. For instance, colonic neurofibromas, perineuriomas and so-called benign fibroblastic polyp of the colon' may all show what is considered to be secondary serrated change. ${ }^{2021}$ Particularly in the right colon, one can see serration in relation to submucosal lipomas. At the current time, it is uncertain whether this is secondary serration

\section{Take home messages}

- The inception of national bowel cancer screening programmes have highlighted serrated colorectal lesions as an area subject in which nomenclature and diagnostic criteria are varied and where the relative risks of disease progression are uncertain.

- We recommend the following nomenclature for serrated colorectal lesions: hyperplastic polyp (HP), sessile serrated lesion (SSL), SSL with dysplasia, traditional serrated adenoma (TSA) and mixed polyp.

- The precise risk of development of colorectal cancer associated with the finding of an SSL and how this might relate to features such as lesion size is currently unclear.

- TSAs are less problematic since, although the minimum diagnostic criteria are still undefined, histopathologists are likely to be able to recognise these lesions as a form of adenoma.

- Most 'mixed polyps' probably represent SSLs with dysplasia. However, we recommend that the term is retained for use in cases where the features favour a true 'collision' lesion.

- Adoption of this guidance should improve interobserver agreement during the histopathological assessment of serrated lesions, facilitate the collection of robust data and lead to a better understanding of the biological significance of these lesions. or whether there is a coincidence of two separate pathologies, namely a lipoma and an SSL.

\section{CONCLUSION}

The key diagnostic difficulties within this spectrum of lesions relate to the differentiation of HPs from SSLs (especially those without dysplasia). ${ }^{22}$ We have made recommendations for the use of a simplified terminology system for serrated lesions. The minimum criteria for diagnosis of SSLs are still the focus of uncertainty and debate. In contrast, TSAs would not usually be mistaken for HPs or SSLs, as the 'classical' dysplasia within them is more immediately obvious. Therefore, the differential diagnosis between 'classical' adenomas and TSAs is of less importance to patient management than the accurate identification of SSLs with and without dysplasia.

\section{Handling editor Cheok Soon Lee}

Acknowledgements This guidance has been discussed within and approved by the pathology committees of the UK Bowel Cancer Screening Programme and the British Society of Gastroenterology. The authors are very grateful to Dr Simon Leedham, Oxford, for helpful discussion of the molecular pathogenesis of serrated pathology of the colorectum.

Contributors $A C B$ and NAS contributed equally to the conceptualisation and preparation of this manuscript. ACB prepared the figures.

Competing interests None declared.

Provenance and peer review Commissioned; externally peer reviewed.

\section{REFERENCES}

1 O'Brien M, Zhao Q, Yang S. Colorectal serrated pathway cancers and precursors. Histopathology 2015;66;49-65.

2 Wong NA, Hunt LP, Novelli MR, et al. Observer agreement in the diagnosis of serrated polyps of the large bowel. Histopathology 2009;55:63-6.

3 Ensari A, Bilezikci B, Carneiro F, et al. Serrated polyps of the colon: how reproducible is their classification? Virchows Arch 2012;461:495-504.

4 Gill P, Wang LM, Bailey A, et al. Reporting trends of right-sided hyperplastic and sessile serrated polyps in a large teaching hospital over a 4-year period (20092012). J Clin Pathol 2013:66:655-8.

5 Torlakovic E, Snover DC. Serrated adenomatous polyposis in humans. Gastroenterology 1996;110:748-55.

6 Lino H, Jass JR, Simms LA, et al. DNA microsatellite instability in hyperplastic polyps, serrated adenomas and mixed polyps: a mild mutator pathway for colorectal cancer? J Clin Pathol 1999;52:5-9.

7 Bateman AC. Pathology of serrated colorectal lesions. J Clin Pathol 2014;67;865-74.

8 Yang S, Farraye YA, Mack C, et al. BRAF and KRAS mutations in hyperplastic polyps and serrated adenomas of the colorectum: relationship to histology and CpG island methylation status. Am J Surg Pathol 2004;28:1452-9.

9 Snover DC, Ahnen DJ, Burt RW, et al. Serrated polyps of the colon and rectum and serrated polyposis. In: Bosman FT, Carneiro F, Hruban RH, Theise ND, eds. World Health Organisation classification of tumours of the digestive system. 4th edn. Lyon: IARC Press, 2010:160.

10 Rex DK, Ahnen DJ, Baron JA, et al. Serrated lesions of the colorectum: review and recommendations from an expert panel. Am J Gastroenterol 2012;107:1315-29.

11 Carr NJ, Mahajan H, Tan KL, et al. Serrated and non-serrated polyps of the colorectum: their prevalence in an unselected case series and correlation of BRAF mutation analysis with the diagnosis of sessile serrated adenoma. J Clin Pathol 2009;62:516-18.

12 Chetty $R$, Bateman AC, Torlakovic E, et al. A pathologist's survey on the reporting of sessile serrated adenomas/polyps. J Clin Pathol 2014;67:426-30.

13 Glatz K, Pritt B, Glatz D, et al. A multinational, Internet-based assessment of observer variability in the diagnosis of serrated colorectal polyps. Am J Clin Pathol 2007;127:983-45.

14 Snover DC. Update on the serrated pathway to colorectal carcinoma. Hum Pathol 2011:42:1-10.

15 Sandmeier D, Seelentag W, Bouzourene H. Serrated polyps of the colorectum: is sessile serrated adenoma distinguishable from hyperplastic polyp in a daily practice? Virchows Arch 2007;450:613-18. 
16 Bustamente-Balen M, Bernet L, Cano R, et al. Assessing the reproducibility of the microscopic diagnosis of sessile serrated adenoma of the colon. Rev Esp Enferm Dig 2009;101:258-64.

17 Yantiss RK, Oh KY, Chen YT, et al. Filiform serrated adenomas: a clinicopathologic and immunophenotypic study of 18 cases. Am I Surg Pathol 2007;31:1238-45.

18 Davis $\mathrm{H}$, Irshad S, Bansal M, et al. Aberrant epithelial GREM1 expression initiates colonic tumorigenesis from cells outside the stem cell niche. Nat Med 2014;21:62-70.

19 Srivastava A, Redston M, Farraye FA, et al. Hyperplastic/serrated polyposis in inflammatory bowel disease: a case series of a previously undescribed entity. Am I Surg Pathol 2008;32:296-303.
20 Eslami-Varzaneh F, Washington $\mathrm{K}$, Robert ME, et al. Benign fibroblastic polyps of the colon: a histologic, immunohistochemical and ultrastructural study. Am I Surg Pathol 2004;28:374-8.

21 Hornick JL, Fletcher CD. Soft tissue perineuroma: clinicopathologic analysis of 81 cases including those with atypical histologic features. Am I Surg Pathol 2005;29:845-58.

22 Aust DE, Baretton GB, Members of the Working group GI-Pathology of the German Society of Pathology. Serrated polyps of the colon and rectum (hyperplastic polyps, sessile serrated adenomas, traditional serrated adenomas, and mixed polyps) - proposal for diagnostic criteria. Virchows Arch 2010;457:291-7 